\title{
LES PHLÉBOTOMES DU LIBAN INVENTAIRE FAUNISTIQUE
}

\author{
HADDAD N*, LÉGER N.** \& SADEK R. ***
}

Summary: SANDFLIES OF LEBANON: FAUNISTIC INVENTORY

An entomological study was made in order to complete the epidemiological data on leishmaniasis in Lebanon. 19 species of sandflies 113 of the genus Phlebotomus and six of the genus Sergentomyial were found. The list includes a recently described species of the Synphlebotomus subgenus (P. saltiae) and eight other species mentioned for the first time in Lebanon: P. neglectus, P. brevis, P. langeroni, P. balcanicus, S. fallax, S. taizi, S. tiberiadis and S. christophersi. Ecological records of each species and its possible involvment in Leishmania transmission are discussed.

KEY WORDS : sandflies, Phlebotomus, Sergentomyia, Leishmania, Lebanon, ecology.

\section{INTRODUCTION}

E ntouré de pays à forte endémicité leishmanienne, le Liban semblait relativement épargné. Suite à la notification de cas importés et autochtones de leishmanioses cutanées et viscérales (Bitar et Nachman, 1964 ; Nuwayri-Salti et al.,1994 ) des enquêtes épidémiologiques y ont été entreprises. Les parasites responsables ont été isolés et identifiés et leur circulation chez l'Homme et le Chien évaluée (Nuwayri-Salti et al., 1994, 1997 ; Knio et al., 2000 ; Nuwayri-Salti et al., 2000 ; Guerbouj et al., 2001). Cependant, mises à part quelques mentions isolées de divers Phlébotomes (Theodor, 1947 ; Theodor et Mesghali, 1964 ; Léger et al., 1995) le volet vecteurs n'avait pas jusqu'ici été exploré de façon systématique. L'inventaire que nous espérons exhaustif des Phlébotomes du Liban et que nous avons réalisé de 1995 à 1997 vient combler en partie cette lacune.

\footnotetext{
* Faculté de santé publique, Université libanaise, Beyrouth, Liban.

** Faculté de pharmacie, Université de Champagne-Ardenne, 51096 Reims Cedex, France.

${ }^{* * *}$ Faculté des sciences, Université américaine de Beyrouth, Liban. Correspondance : Nabil Haddad, Laboratoire d'immunologie, Faculté de santé publique, Université libanaise, Fanar, Liban.

Tél. : 9613766981 - Fax : 9611691990.

E-mail : nabhaddad@ul.edu.lb
}

\section{Résumé :}

Une étude entomologique a été réalisée afin de compléter les données épidémiologiques concernant la Leishmaniose au Liban. Cette étude a permis d'établir un inventaire faunistique révélant la présence de 19 espèces: 13 du genre Phlebotomus et six du genre Sergentomyia. Parmi elles, une espèce du genre Synphlebotomus (P. saltiae) nouvelle pour la science ainsi que huit espèces jamais signalées au Liban : P neglectus, P. brevis, P. langeroni, P. balcanicus, S. fallax, S. taizi, S. tiberiadis et S. christophersi. Les conditions écologiques de chaque espèce et leur éventuel rôle dans la transmission des Leishmanioses sont discutés dans ce travail.

MOTS CLÉS : phlébotomes, Phlebotomus, Sergentomyia, Leishmania, Liban, écologie.

\section{MATÉRIELS ET MÉTHODE}

T es captures ont été réalisées selon trois techniques :

11 - La technique des pièges adhésifs : des feuilles blanches enduites d'huile de ricin ont été posées régulièrement et laissées pendant trois jours entre mai et septembre 1995. Ces pièges ont été placés le long de 16 itinéraires-transects comportant plus de 200 stations (carte 1) explorant des biotopes aussi variés que possible. En 1996, un itinéraire-transect de 28 stations (carte 2) a été piégé une fois tous les 15 jours entre mai et octobre afin d'observer la dynamique des populations des différentes espèces présentes et de capturer celles à apparition irrégulière dans la saison. Dans chaque station, les pièges ont été posés puis ramassés après la troisième nuit. 2 - La technique des pièges lumineux : les pièges de type $\mathrm{CDC}^{1}$ miniature ont été posés au cours des étés 1995, 1996 et 1997 dans différents habitats (chambres à coucher, bergeries, poulaillers, jardins privés,...) dans diverses localités (carte 2 ).

3 - La capture nocturne manuelle à l'aide d'un aspirateur sur murs et appâts humains. Cette technique a été très rarement appliquée.

\footnotetext{
${ }^{1}$ John W. Hock Company. Insect sampling devices for medical, veterinary and agricultural entomology, P.O.box 12852, Gainesville, FL 32604, USA
} 


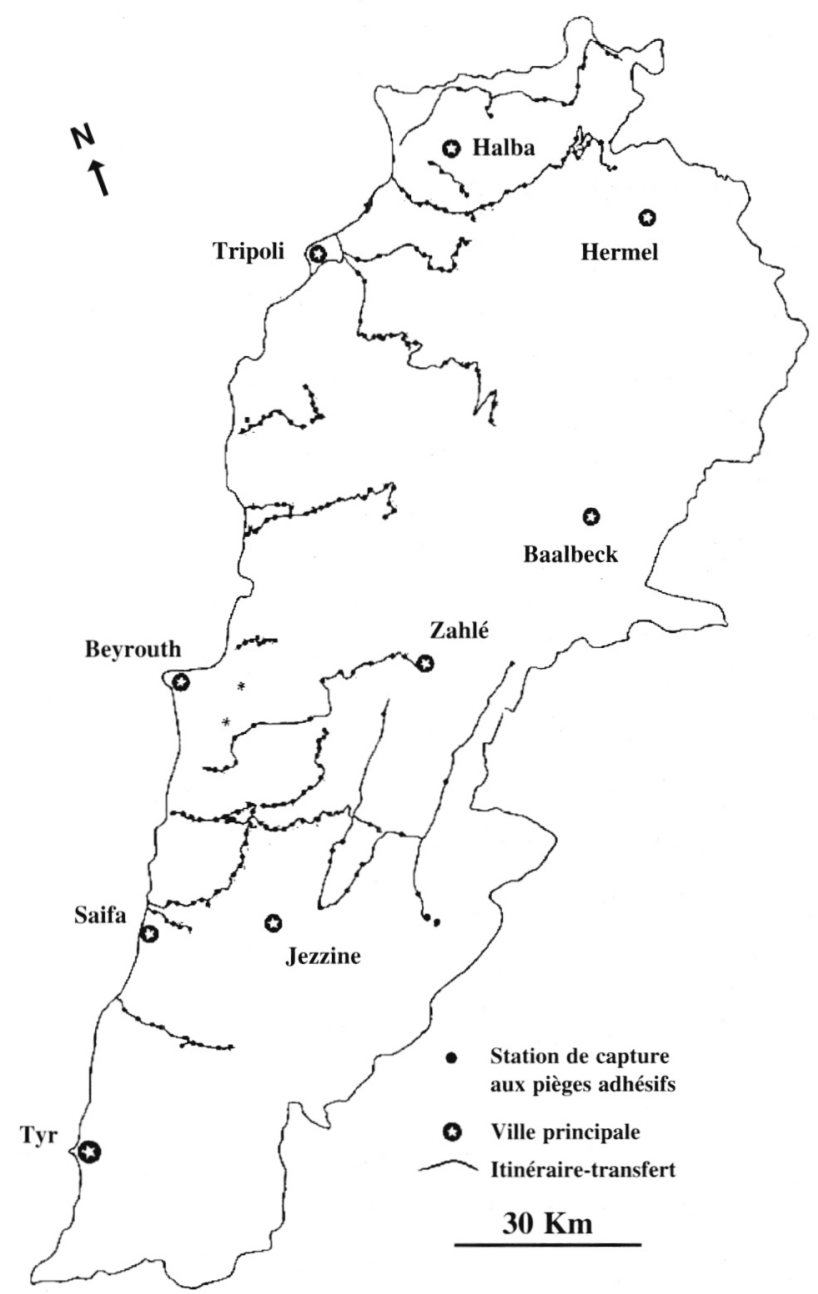

Carte 1. - Itinéraires-transects réalisés pour l'étude chronologique.

\section{RÉSULTATS GLOBAUX}

1 3,570 spécimens de phlébotomes appartenant à 19 espèces ont été capturés : 13 du genre Pblebotomus et six du genre Sergentomyia (tableau I). $S$. dentata est de loin l'espèce la plus abondante dans nos captures $(95,96 \%$ de l'ensemble des spécimens capturés). Au sein du genre Phlebotomus, qui comporte la quasi totalité des espèces anthropophiles et donc d'intérêt médical, P. syriacus constitue l'espèce dominante (49 \% des Phlebotomus) suivi par P. tobbi $(13,20 \%)$. Les autres espèces sont moins fréquentes, voire exceptionnelles, telles $P$. langeroni, S. christophersi et $S$. palestinensis ne figurant qu'à un seul exemplaire dans notre inventaire.

L'analyse des spécimens a révélé la présence d'espèces jamais signalées au Liban : P. neglectus, P. brevis, $S$. fallax, S. taizi, S. tiberiadis et $S$. christophersi, voire même, à l'est de la méditerranée : P. langeroni, $P$. balcanicus. P. (Synphlebotomus) saltiae Léger et al., 1997 a été décrite suite à cette enquête.

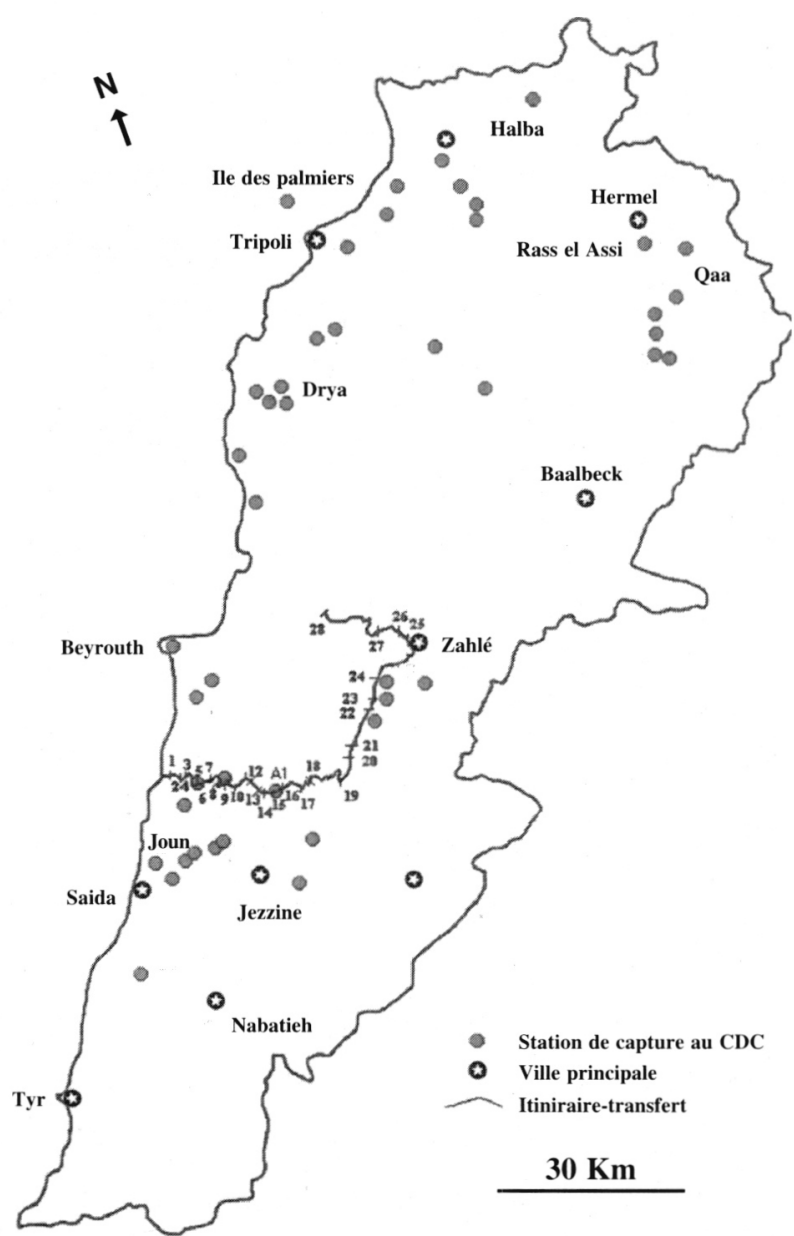

Carte 2. - Stations de piégages aux CDC et itinéraire-transect réalisé pour l'étude chronologique.

Si l'on considère la capture au Liban de P. kandelakii par Theodor et Mesghali (1964) et par Léger et al. (1995), et de P. arabicus (Léger, non publié), le nombre d'espèces trouvées au Liban s'élève à 21 .

\section{COMMENTAIRES SUR LES ESPÈCES}

\section{GenRe PhLEBOtomus}

\section{SOUS-genRe PHLEBOTOMUS}

- Phlebotomus (Phlebotomus) papatasi (Scopoli, 1786) Presque tous les $P$. papatasi ont été capturés uniquement par la méthode des pièges lumineux. La majorité des spécimens capturés provient de l'enclave aride située au nord de la région de Hermel (carte 2), considérée comme le prolongement de la zone aride qui traverse la Syrie dans la direction nord-sud en passant par les régions d'Alep, Hama et Homs. Cette espèce a surtout été capturée dans les bergeries et les étables ainsi que dans les poulaillers. 


\begin{tabular}{|c|c|c|c|c|c|c|c|c|c|c|c|}
\hline \multirow{5}{*}{\begin{tabular}{|c|} 
Effectif total \\
Genre \\
Effectif \\
Type de piège
\end{tabular}} & \multicolumn{11}{|c|}{43570} \\
\hline & \multicolumn{5}{|c|}{ Sergentomyia } & \multicolumn{6}{|c|}{ Pblebotomus } \\
\hline & \multicolumn{5}{|c|}{41858} & \multicolumn{6}{|c|}{1712} \\
\hline & \multicolumn{2}{|c|}{ Adhésifs } & \multicolumn{2}{|c|}{ CDC } & \multirow[b]{2}{*}{ Total } & & \multicolumn{2}{|c|}{ Adhésifs } & \multicolumn{2}{|c|}{ CDC } & \multirow[b]{2}{*}{ Total } \\
\hline & $\delta$ & q & $\delta$ & q & & & $\delta$ & q & o & q & \\
\hline S. (Serg) dentata & 12461 & 28784 & 233 & 328 & 41806 & P. (Phleb) papatasi & 1 & & 112 & 76 & 189 \\
\hline S. (Serg) taizi & 4 & 7 & & 1 & 12 & P. (Larr) syriacus & 290 & 104 & 164 & 281 & 839 \\
\hline S. (Serg) fallax & & 2 & & & 2 & P. (Larr) neglectus & & 6 & & & 6 \\
\hline S. (Sint) tiberiadis & 16 & 6 & 8 & 6 & 36 & P. (Larr) tobbi & 32 & 8 & 61 & 125 & 226 \\
\hline S. (Sint) christophersi & & 1 & & & 1 & P. (Larr) langeroni & & & 1 & & 1 \\
\hline \multirow[t]{8}{*}{ S. (Parrot) palestinensis } & & 1 & & & 1 & P. (Para) jacusieli & 41 & 27 & 35 & 40 & 143 \\
\hline & & & & & 41858 & P. (Para) alexandri & & & 6 & 13 & 19 \\
\hline & & & & & & P. (Para) sergenti & & & 6 & 9 & 15 \\
\hline & & & & & & P. (Ad) simici & 21 & $7^{*}$ & 34 & $124^{*}$ & 186 \\
\hline & & & & & & P. (Ad) brevis & 10 & & 2 & $15^{* *}$ & 27 \\
\hline & & & & & & P. (Ad) balcanicus & & & 5 & $8^{*}$ & 13 \\
\hline & & & & & & P. (Trans) canaaniticus & 2 & 2 & 13 & 20 & 37 \\
\hline & & & & & & P. (Syn) saltiae & & 1 & 3 & 7 & 11 \\
\hline
\end{tabular}

* Femelles du sous-genre Adlerius, leur détermination spécifique n’a pas été possible selon les critères morphologiques.

** femelles déterminées par la méthode enzymatique.

Tableau I. - Liste des espèces trouvées.

\section{SOUS-GENRE LARROUSSIUS}

- Phlebotomus (Larroussius) syriacus Adler et Theodor, 1931 (figure 1)

$P$. syriacus constitue dans nos captures l'espèce dominante du genre Phlebotomus. Présente dans toutes les zones prospectées au Liban, elle est plus abondante en moyenne altitude ( 400 à $800 \mathrm{~m}$ ) et dans les stations relativement humides. Elle a été capturée à l'intérieur des habitations ainsi que dans les étables. Son rôle dans la transmission de Leishmania infantum, souvent évoqué, n’a pas été jusqu'ici prouvé. Adler et Theodor (1931) disent l'avoir infesté expérimentalement, mais à la lumière des récentes mises au point taxinomiques (Léger et Pesson, 1987), il s'agissait probablement de l'espèce affine: $P$. neglectus.
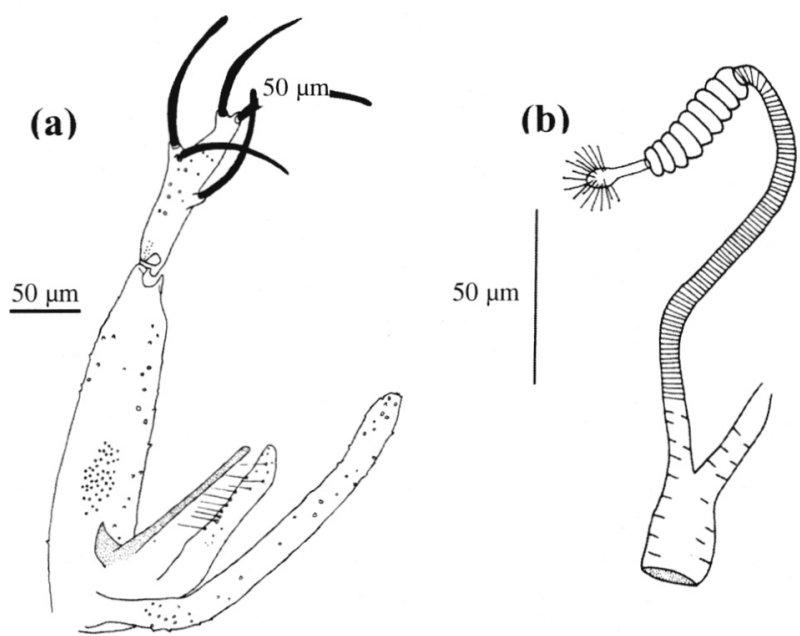

Fig. 1. - P. syriacus. Génitalia mâle (a); spermathèque (b).
- Phlebotomus (Larroussius) neglectus Tonnoir, 1921

La femelle de $P$. neglectus se distingue de celle de $P$. syriacus par l'étendue de l'armature pharyngienne dépassant la moitié de la partie élargie du pharynx, alors que chez $P$. syriacus elle occupe son tiers inférieur (figure 2). À notre avis, ce caractère justifie le maintien de ces deux taxons par Léger et Pesson (1987) après leur mise en synonymie par Artemiev et Neronov en 1984.

Il nous a été impossible de séparer les mâles de $P$. neglectus de ceux de P. syriacus. Le nombre de soies

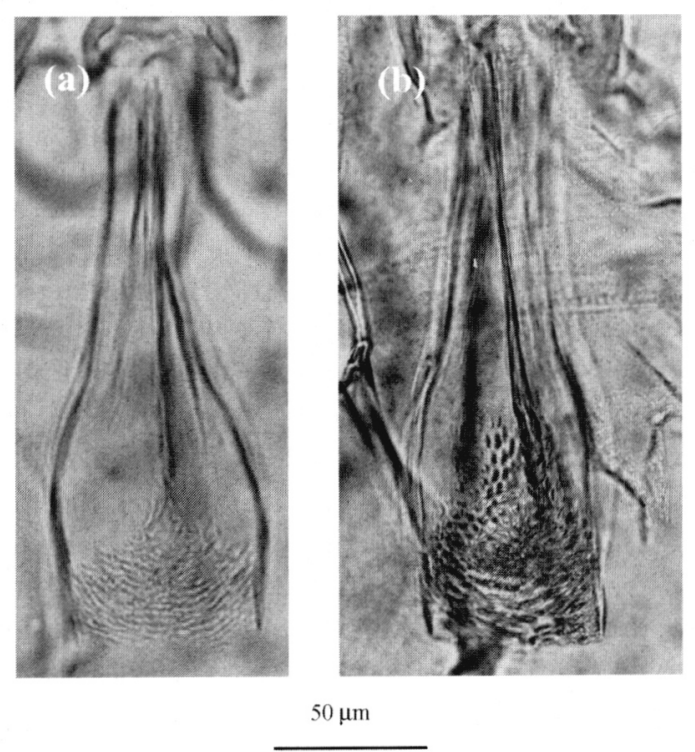

Fig. 2. - Armature pharyngienne femelle : P. syriacus (a ; P. neglectus (b). 


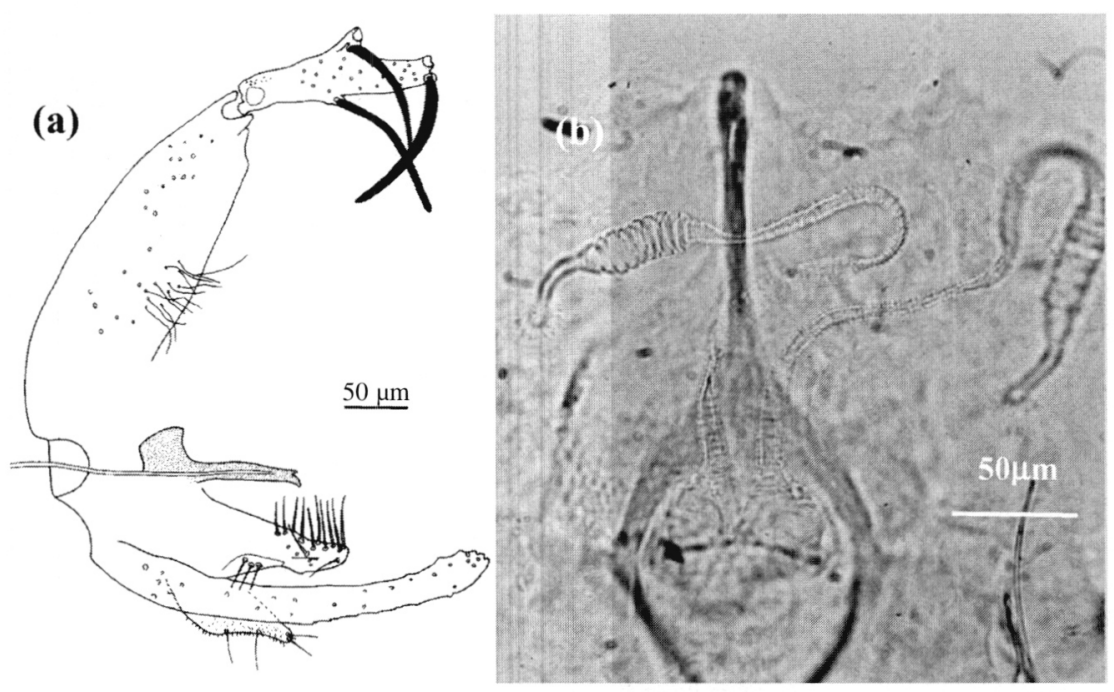

Fig. 3. - P. tobbi. Génitalia mâle (a) ; spermathèques (b).

du coxite est parfois proposé comme caractère de différenciation spécifique (Léger et Pesson, 1987) : 20 à 30 soies pour $P$. neglectus, alors que ce nombre est plus élevé chez P. syriacus et atteint la soixantaine. Au Liban, ce caractère ne semble pas être valable du fait que dans les zones où des femelles de type neglectus n'ont pas été trouvées, $30 \%$ des mâles capturés ont un nombre de soies compris entre 20 et 30 .

Seulement six femelles capturées à Rass el Assi (Hermel) possèdent une armature pharyngienne importante de type neglectus. Leur présence, en sympatrie avec $P$. syriacus dans cette région du nord de la Bekaa ainsi qu'au nord-ouest de la Syrie (Ismail et Pesson, 1992), nous laisse penser que la zone de recouvrement de leurs aires de répartition respectives se situent à ce niveau. Notons que $P$. neglectus est un vecteur confirmé de leishmaniose viscérale en Grèce (Léger et al., 1988). Rare au Liban, son rôle y est peu probable.

- Phlebotomus (Larroussius) tobbi Adler, Theodor et Lourie, 1930 (figure 3)

P. tobbi est mieux représenté dans nos captures aux pièges CDC que dans celles aux pièges adhésifs. À Qabaait (région de Akkar), elle est abondante dans les étables où elle constitue plus de $30 \%$ des captures. Toutefois, la présence d'hématies nucléées chez les femelles gorgées laisse à penser qu'elle se nourrit sur les volailles. La morphologie des spécimens observés au Liban est comparable à celle décrite de Grèce (Léger et al., 1986 ; Madulo-Leblond, 1983) et de Syrie (Killick-Kendrick et al., 1991). Cependant, une certaine variabilité au niveau de la morphologie des dilatations des conduits des spermathèques a été observée sur certains spécimens provenant de Qabaait : asymétrie plus accusée, plis plus marqués qui les rapprochent de celles observées chez $P$. perfiliewi. Des variations similaires ont été également observées en Grèce, à Rhodes et à Chypre (Depaquit et al., 2001) ainsi qu'en Syrie (observation personnelle).
Cette espèce pourrait jouer un rôle essentiel dans la transmission de la leishmaniose viscérale au Liban d'autant que des femelles ont été trouvées naturellement infestées par Leishmania donovani en Syrie (Rioux et al., 1998) et par L. infantum à Chypre (Léger et al., 2000). Elle a été capturée le long du versant est du Mont Liban jusqu'à une altitude de 900 mètres.

- Phlebotomus (Larroussius) langeroni Nitzulescu, 1930 (figure 4)

Un seul spécimen mâle, associé à $P$. papatasi et $S$. dentata, a été trouvé à Qaa (région de Hermel) dans un

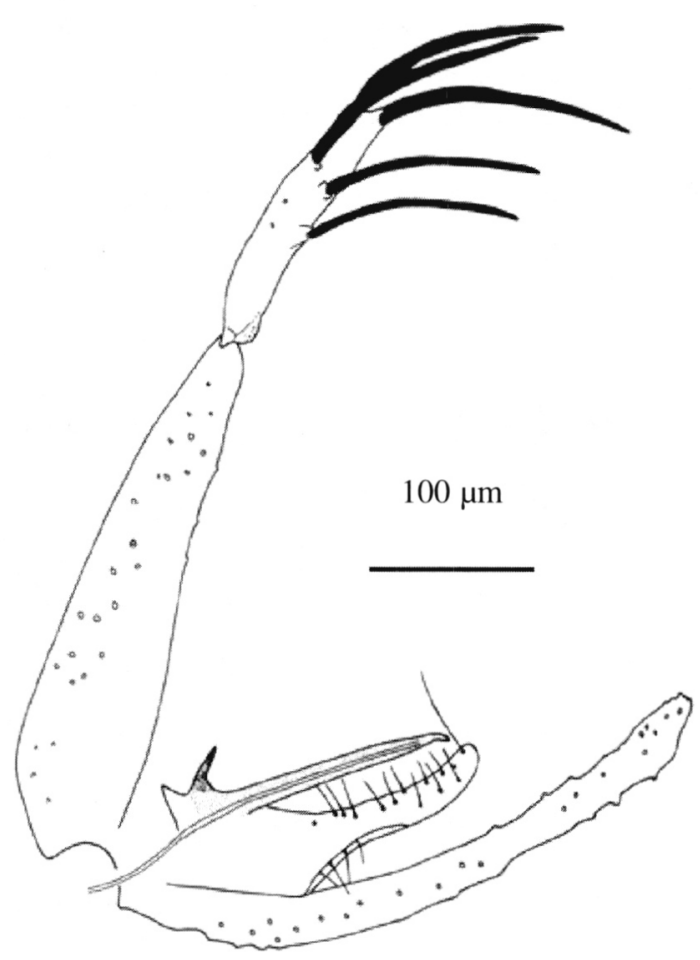

Fig. 4. - P. langeroni. Génitalia mâle. 
piège lumineux. Sa présence au Liban constitue une extension septentrionale de son aire de répartition limitée jusque là à l'Afrique du nord et l'Égypte. Son pouvoir vecteur a été confirmé dans le foyer d'Al Agamy (Doha et Shehata, 1992). Notre diagnose repose sur les caractères suivants : valves copulatrices en forme de cône allongé, taillées en biseau court se terminant par une seule pointe dirigée vers l'intérieur ; troisième segment antennaire long (AII > AIV + AV) ; épines géniculées assez longues (celles de AIV mesurent un peu moins du tiers de la longueur de l'article) ; formule antennaire 2/III-XIII.

\section{SOUS-GENRE ADLERIUS}

L'identification des espèces de ce sous-genre se fait sur des spécimens mâles. Les femelles sont réputées indifférenciables sur la base des caractères morphologiques.

- Pblebotomus (Adlerius) simici Nitzulescu, 1931 (figure 5) Présent sur tout le versant ouest du Mont Liban, où sa densité diminue en allant vers le sud, on le trouve surtout à une altitude moyenne (400-800 m) correspondant à l'étage euméditerranéen à $Q$. calliprinos et $Q$. infectoria. La majorité des spécimens capturés provient essentiellement de Drya (région de Jbeil), Danbo et de Qabaait (région de Akkar).

- Phlebotomus (Adlerius) brevis Theodor et Mesghali, 1964 (figure 6)

$P$. brevis a été capturé en milieu rural, essentiellement dans les étables et les bergeries, aussi bien aux pièges adhésifs qu'aux pièges CDC, dans des stations de moyenne et haute altitude sur le versant est du Mont

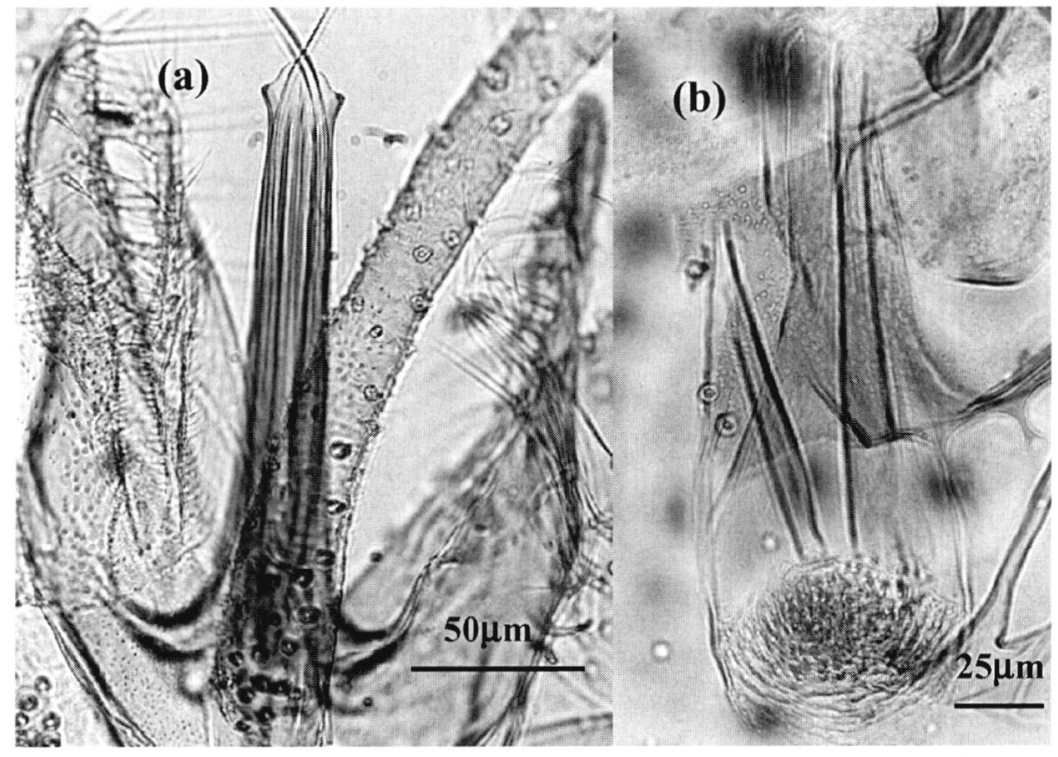

Fig. 5. - P. simici. Mâle : édéage (a); femelle : armature pharyngienne caractérisant le sousgenre (b).
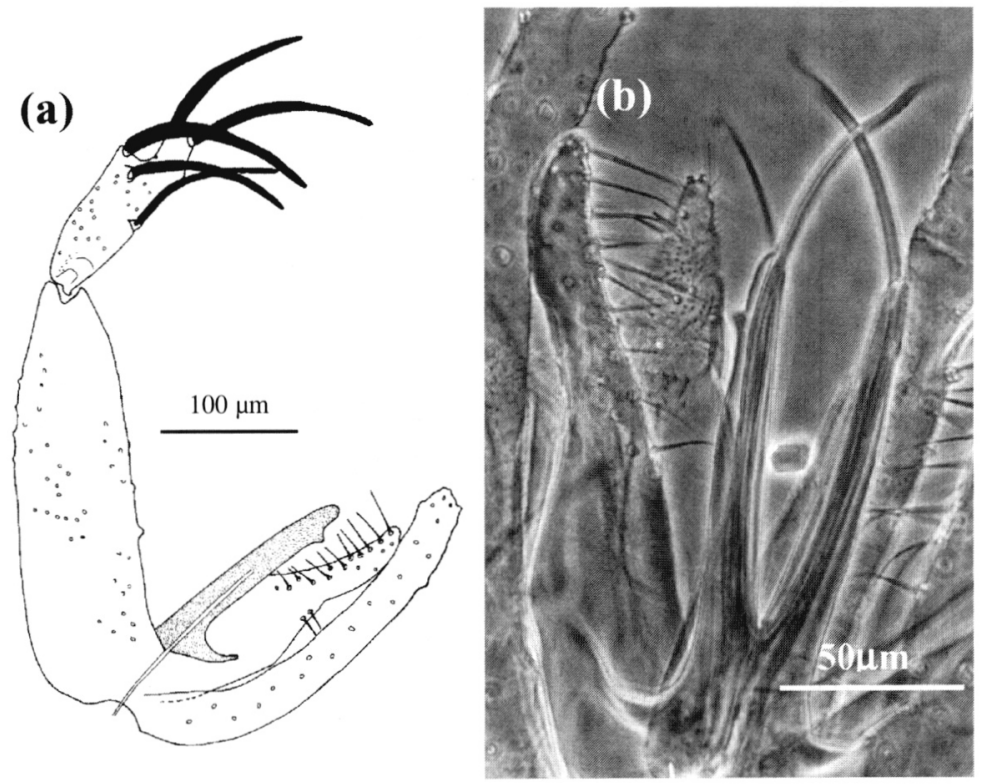

Fig. 6. - P. brevis. Mâle : génitalia (a); édéage (b). 
Liban atteignant $2070 \mathrm{~m}$ au niveau de la station d'AlArz (altitude la plus élevée pour les Phlébotomes du Liban).

La différenciation entre $P$. brevis et $P$. simici se fait classiquement sur les spécimens mâles. Les femelles restent indifférenciables malgré la révision d'Artemiev en 1981. Cependant, selon une étude réalisée par Perrotey et al. (2000) sur des spécimens provenant de Drya et de Danbo, les femelles de $P$. simici fraîchement disséquées auraient des réservoirs plus courts que ceux de $\mathrm{P}$. brevis et les bases des spermathèques plus longues.

- Pblebotomus (Adlerius) balcanicus Theodor, 1958 (figure 7)

Cinq individus mâles de $P$. balcanicus et huit femelles d'Adlerius ont été trouvés dans une ferme à Rass el Assi (Hermel). Cette localité est caractérisée par un climat aride et une végétation basse rare de type steppique sur sol caillouteux calcaire. La diagnose spécifique s'appuie sur deux types de critères :
- critères morphologiques, en particulier le nombre et la disposition de soies du coxite conformes à la description donnée par Dancesco (1968) et Artemiev (1980) ;

- critères biogéographiques basés sur l'aire de répartition connue de $P$. balcanicus atteignant les pays voisins du Liban (nord de la Grèce et la Turquie).

\section{SOUS-GENRE SYNPHLEBOTOMUS}

- Phlebotomus (Synphlebotomus) saltiae Léger, Haddad et Chaker, 1997 (figure 8)

Cette espèce constitue la première mention du sous genre Synphlebotomus au Proche-Orient. Elle a été décrite de Choueifat, à coté de Beyrouth, et retrouvée dans trois localités relativement éloignées les unes des autres : Joune, Deir Dourite et Markabta. P. saltiae semble être une espèce des zones humides et semiarides. Elle a été également capturée en milieu cavernicole.
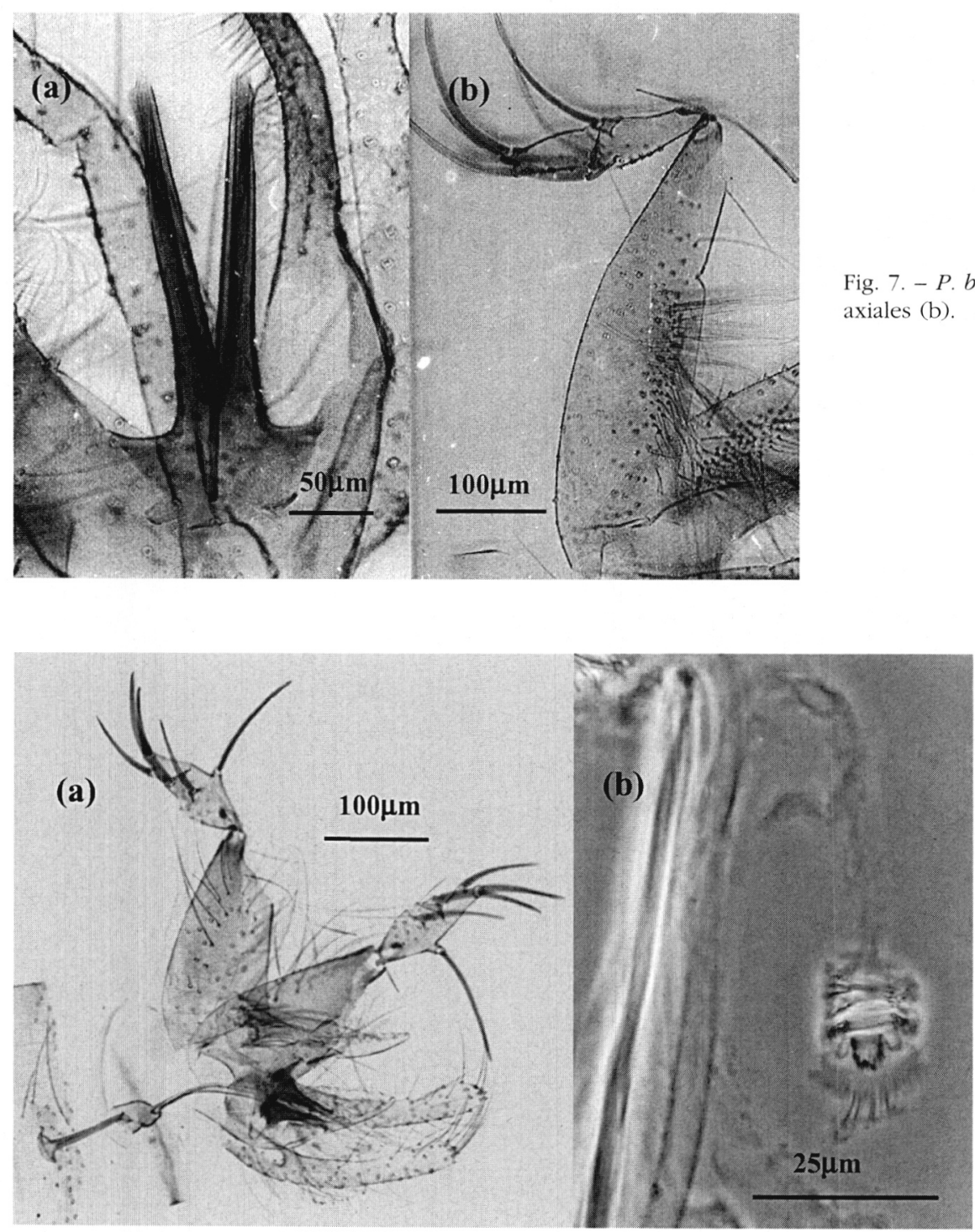

Fig. 7. - P. balcanicus. Édéage (a) ; plaque de soies coaxiales (b)
Fig. 8. - P. saltiae. Génitalia mâle (a); spermathèque (b). 


\section{SOUS-GENRE PARAPHLEBOTOMUS}

- Phlebotomus (Paraphlebotomus) jacusieli Theodor, 1947 (figure 9)

$P$. jacusieli est relativement abondant dans nos captures : c'est l'espèce de Paraphlebotomus la mieux représentée. Sa répartition au Liban est assez vaste ; elle concerne la plaine de la Bekaa ainsi que le versant ouest du Mont Liban, depuis le niveau de la mer jusqu'à $1250 \mathrm{~m}$ (Ehden), avec une abondance plus marquée en moyenne altitude $(500-900 \mathrm{~m})$.

Cette espèce a été trouvée dans des zones humides et subhumides où les précipitations annuelles sont supérieures à $600 \mathrm{~mm}$. C'est le seul Paraphlebotomus présent dans ce type de biotope. Elle est fréquente dans les poulaillers. Nous l'avons également capturé dans des étables et des bergeries.

- Phlebotomus (Paraphlebotomus) sergenti Parrot, 1917 (figure 10)

La morphologie des spécimens trouvés au Liban répond aux critères d'identification de $P$. sergenti $\mathrm{s}$. str., vecteur prouvé de Leishmania tropica au Moyen-Orient (Al-Zahrani et al.,1988), et non pas à ceux de P. similis (Depaquit, 1997). Selon nos captures, cette espèce paraît peu abondante. Sa distribution reste confinée aux zones arides $(\mathrm{P} \leq 200 \mathrm{~mm} / \mathrm{an})$ du nord de la Bekaa (El Qaa).

- Phlebotomus (Paraphlebotomus) alexandri Sinton, 1928 (figure 11)

Cette espèce est le vecteur prouvé de L. infantum en Chine occidentale (Guan et al., 1986). En outre son rôle dans la transmission de la leishmaniose cutanée est fortement suspecté dans certains foyers comme l'Iran et la Tunisie (Javadian et al., 1977 et Croset et al., 1978). P. alexandri apparaît au Liban comme une espèce des zones arides. Elle a été seulement capturée dans l'enclave aride au nord de la Bekaa à Rass el Assi, Qaa et Aarsal (région de Hermel) où la pluviométrie ne dépasse pas $400 \mathrm{~mm}$ par an. De même, nous l'avons

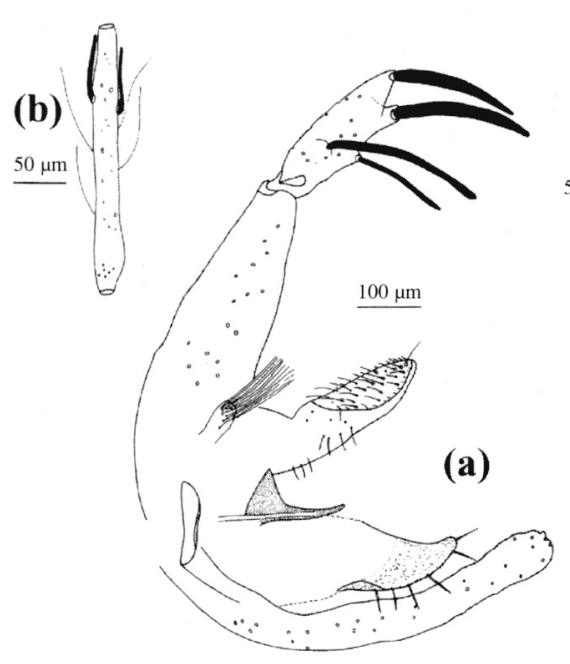

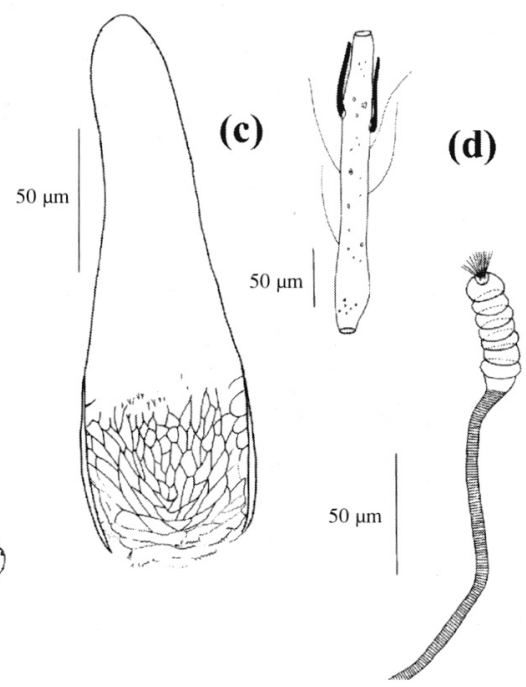

(e) Fig. 9. - P. jacusieli. Mâle : génitalia (a) segment antennaire $A_{\text {III }}$ (b). Femelle : pharynx (c) ; segment antennaire (d) ; spermathèque (e).

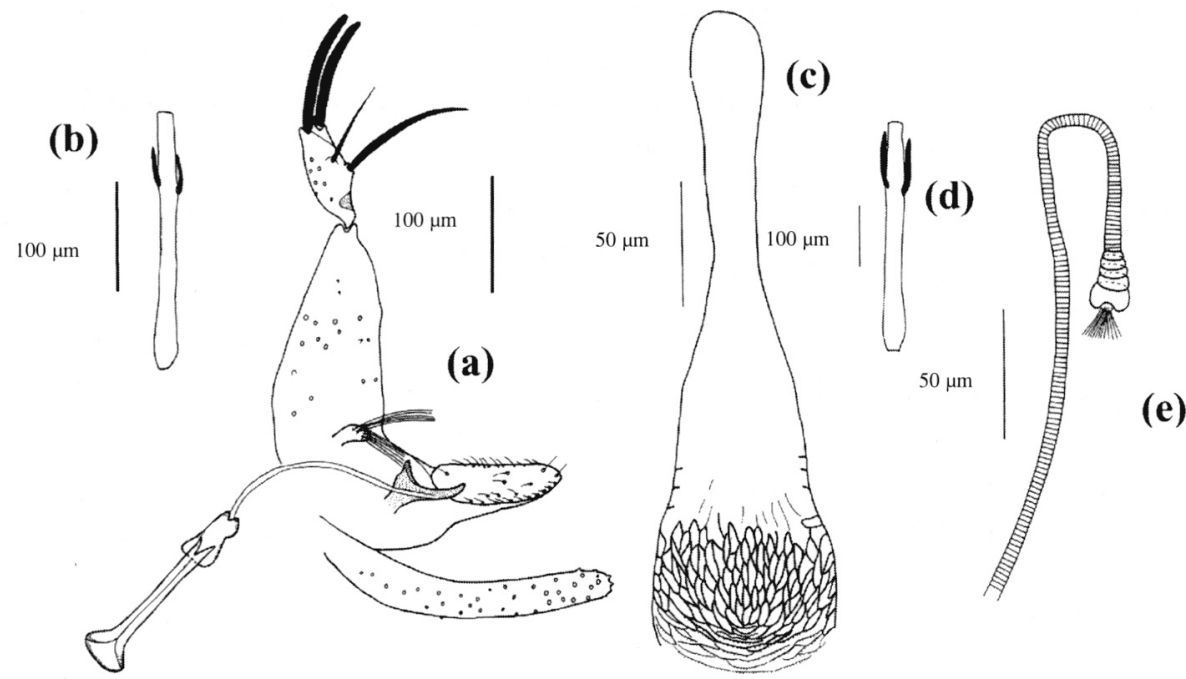

Fig. 10. - P. sergenti. Mâle : génitalia (a); segment antennaire $A_{\text {III }}$ (b). Femelle : pharynx (c) ; segment antennaire (d) ; spermathèque (e). 

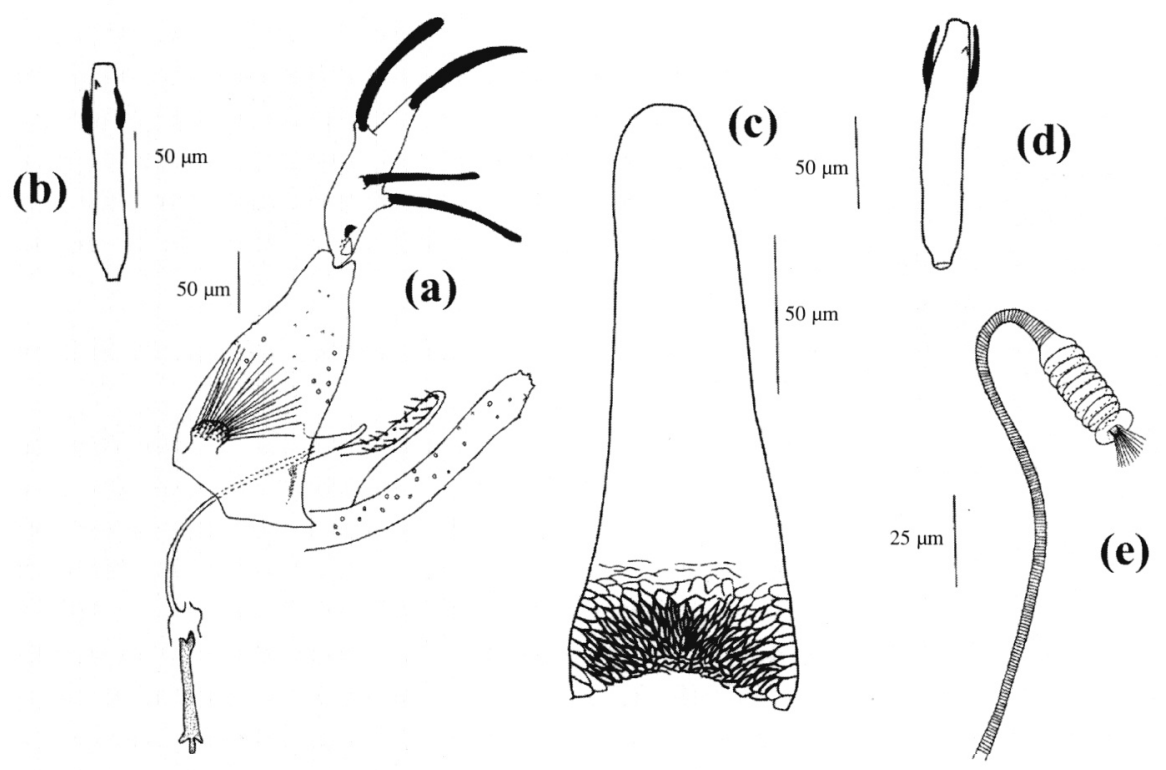

Fig. 11. - P. alexandri. Mâle : génitalia (a); segment antennaire $A_{\mathrm{III}}$ (b). Femelle : pharynx (c) ; segment antennaire (d) ; spermathèque (e).

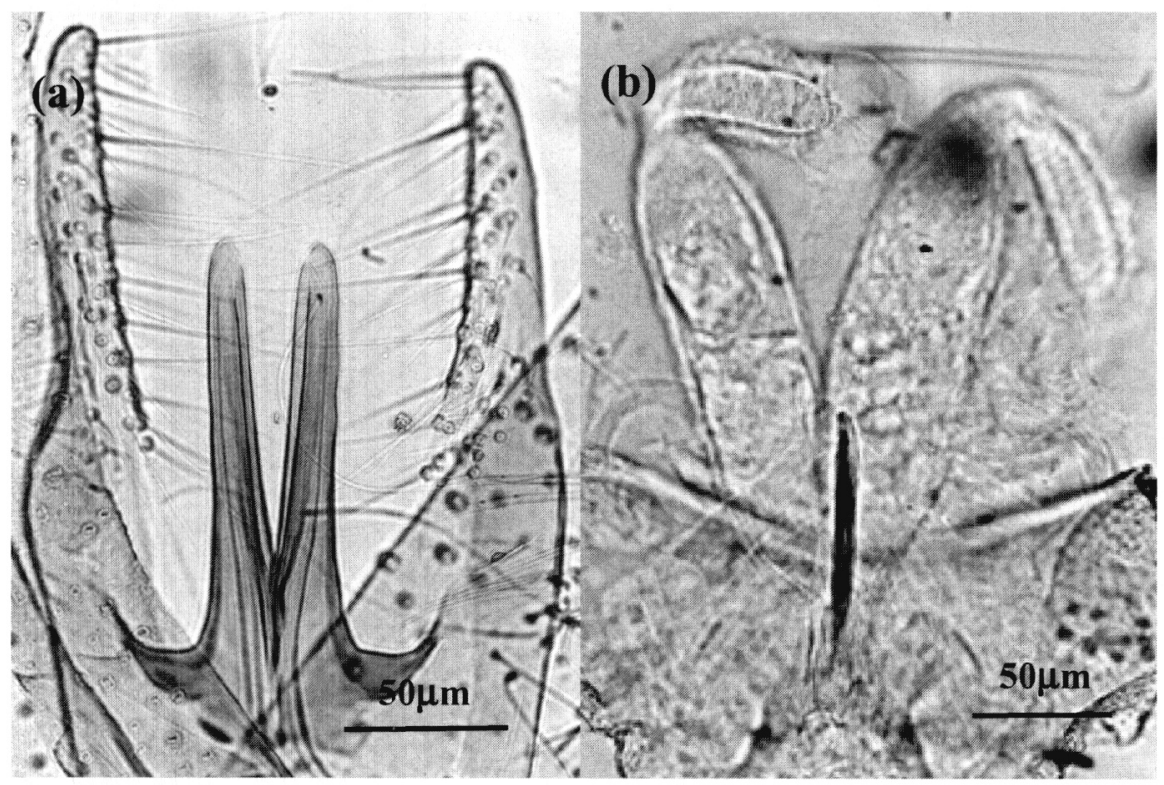

Fig. 12. - P. canaaniticus. Édéage (a); spermathèque $(b)$

également capturé en Syrie, dans la région aride de Rhaibé

\section{SOUS-GENRE TRANSPHLEBOTOMUS}

- Phlebotomus (Transphlebotomus) canaaniticus Adler et Theodor, 1931 (figure 12)

Le sous genre Transphlebotomus renferme actuellement trois espèces : $P$. canaaniticus, $P$. mascittii et $P$. economidesi. Cette dernière a été décrite de Chypre et se différencie des deux autres espèces par la morphologie des spermathèques. En revanche, les femelles de $P$. mascittii et $P$. canaaniticus sont difficiles à séparer. L'utilisation des critères biométriques permet de confirmer que l'espèce trouvée au Liban est $P$. canaaniticus.

33 spécimens ont été capturés essentiellement par des pièges lumineux dans des stations à climat humide et végé- tation dense : Baaqline $(750 \mathrm{~m})$ et Ouadi Fissane $(1100 \mathrm{~m})$. Quatre femelles ont été récoltées à Rass el Assi dans l'enclave aride. Notons que dans les captures effectuées en milieu cavernicole (Wadi Jihanam), cette espèce est dominante et représentée essentiellement par des mâles.

\section{GENRE SERGENTOMYIA}

SOUS-GENRE SERGENTOMYIA

- Sergentomyia (Sergentomyia) dentata (Sinton, 1933) (figure 13)

S. dentata est de loin l'espèce dominante dans les captures réalisées par la technique des pièges adhésifs. Elle constitue plus de $98 \%$ des spécimens récoltés. Sa distribution au Liban est très large. Elle a été trouvée dans la plupart des stations prospectées. 

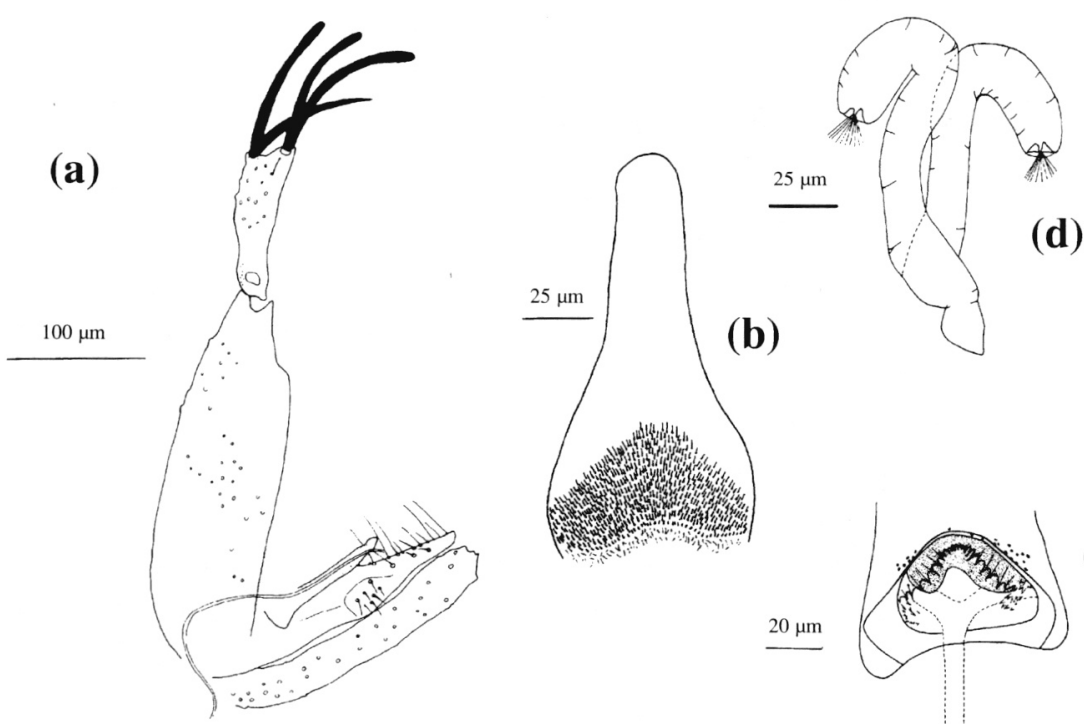

Cette espèce semble tolérer les différents biotopes puisqu'elle est abondante aussi bien dans les zones semi-arides (la Bekaa) que dans les zones humides (Deir Dourite), colonisant les différents étages altitudinaux du Mont Liban depuis le niveau de la mer jusqu'à $1750 \mathrm{~m}$.

Un grand polymorphisme existe au sein de la population proche-orientale. La femelle de $S$. dentata possède un cibarium armé de 16 à 30 dents disposées sur une ligne courbe. Les dents médianes sont nettement plus petites que les latérales. Une étude morphométrique portant sur le nombre de dents cibariales chez la population libanaise de $S$. dentata montre qu'il s'agit d'une seule population, tout au moins en ce qui concerne le caractère étudié.

- Sergentomyia (Sergentomyia) fallax (Parrot, 1921) (figure 14)

Seulement deux femelles de cette espèce ont été capturées sur des pièges adhésifs durant cette étude : une à Dmitt $(250 \mathrm{~m})$ et une autre à Zahlé $(1400 \mathrm{~m})$. La rareté de cette espèce peut être expliquée par son inféodation au climat désertique.

- Sergentomyia (Sergentomyia) taizi Lewis, 1974 (figure 15) 12 spécimens (huit femelles, quatre mâles) de S. taizi ont été récoltés dans trois stations (Dmitt, Multaka-elNahraïne et à Almane) dont d'altitude ne dépasse pas $300 \mathrm{~m}$, et qui appartiennent à l'étage thermo-méditerranéen représenté par la série de végétation du caroubier-lentisque.

\section{SOUS-GENRE SINTONIUS}

- Sergentomyia (Sintonius) tiberiadis (Adler, Theodor et Lourie, 1930) (figure 16)

Contrairement aux observations de Büttiker et Lewis (1983) en Arabie Saoudite, les spécimens capturés prothèques (c) prinos. (figure 17)
Fig. 13. - P. dentala. Mâle : génitalia (a). Femelle : pharynx (b) ; cibarium (c); spermathèques $(\mathrm{d})$.

\section{(c)}
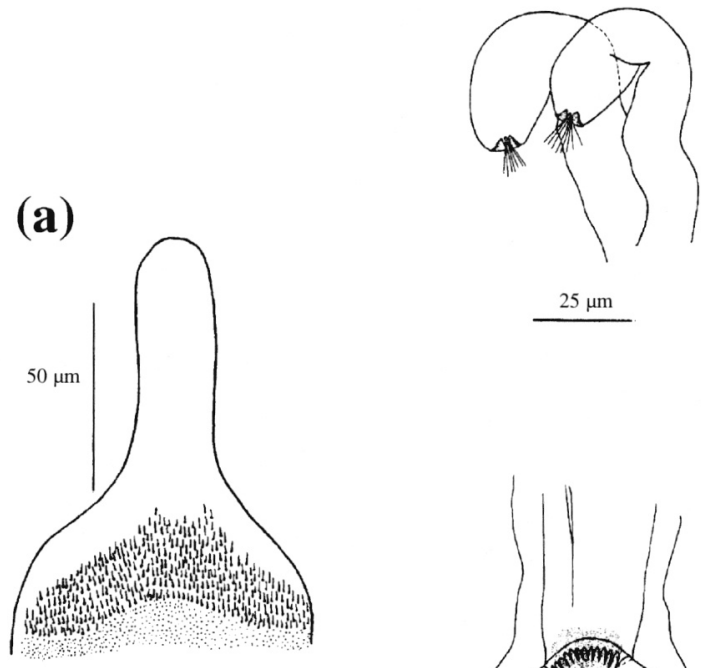

(c)

Fig. 14. - S. fallax. Femelle : pharynx (a) ; cibarium (b) ; sperma-

viennent des stations de basse altitude : du niveau de la mer (Damour) jusqu'à $400 \mathrm{~m}$ (Jahlieh). Ceci correspond essentiellement à l'étage thermo-méditerranéen, relativement humide et chaud peuplé par les séries du caroubier-lentisque et du Quercus calli-

- Sergentomyia (Sintonius) christophersi (Sinton, 1927)

Un seul spécimen femelle a été capturé à Almane (150 m), à l'étage thermo-méditerranéen, dans une anfractuosité rocheuse sur substrat calcaire. 


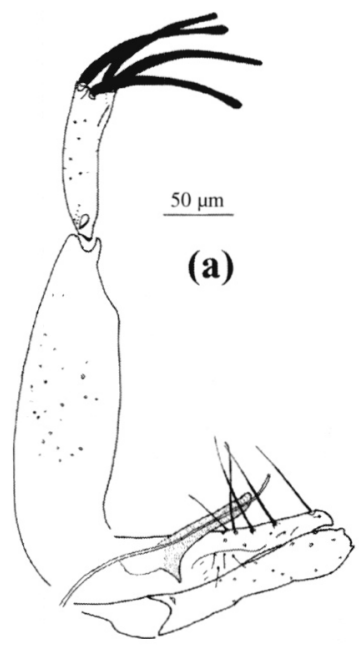

(b)
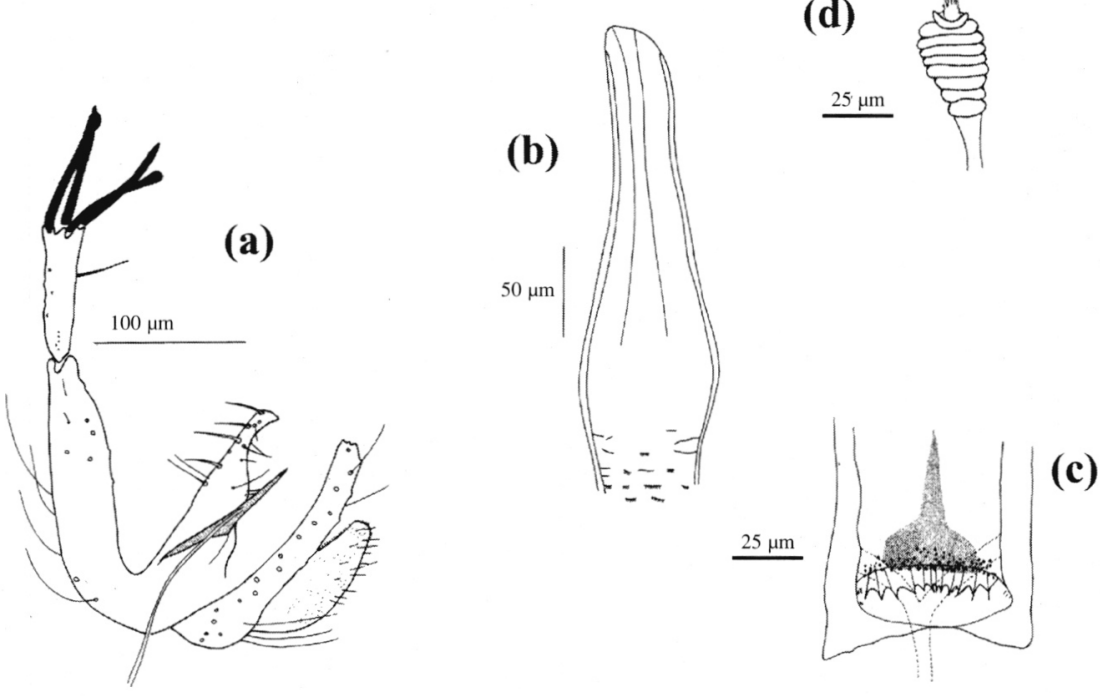

Fig. 15. - S. taizi. Mâle : génitalia (a). Femelle : pharynx (b) ; cibarium (c) ; spermathèques $(\mathrm{d})$.
Fig. 16. - S. tiberiadis. Mâle : génitalia (a). Femelle : pharynx (b) ; cibarium (c) ; spermathèques $(\mathrm{d})$.

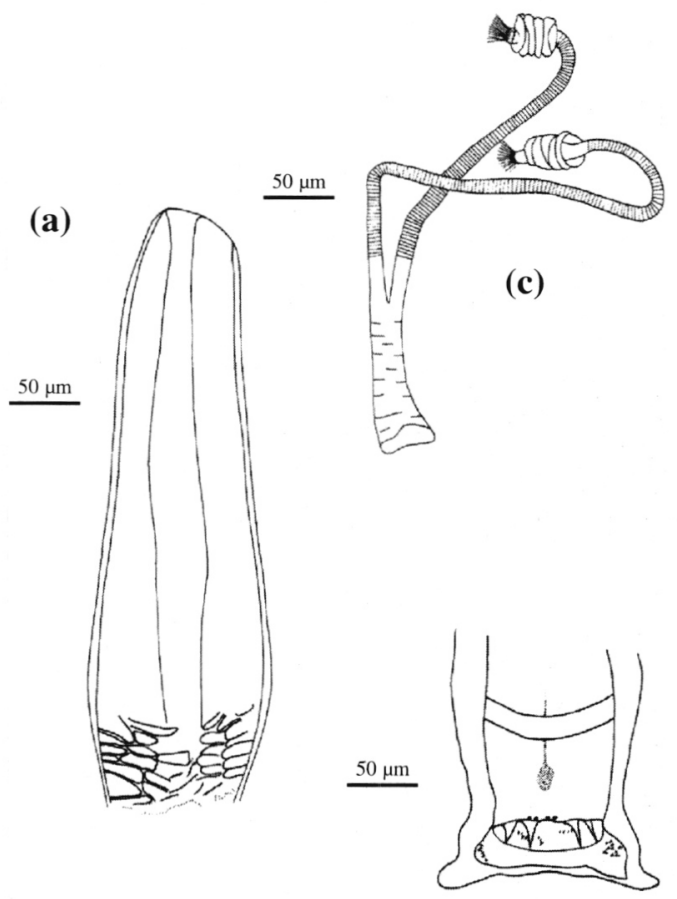

SOUS-GENRE PARROTOMYIA

- Sergentomyia (Parrotomyia) palestinensis (Adler et Theodor, 1927) (figure 18)

Un seul spécimen femelle a été capturé à Choueïfat au sud-est de Beyrouth.

\section{CONCLUSION}

T 'inventaire réalisé a permis d'identifier 19 espèces de Phlébotomes. La plupart des espèces réperto1 riées sont paléarctiques, certaines sont d'affinités africaines ( $P$. saltiae et $S$, taizi), ce qui témoigne de l'importance de la région en tant que couloir de peuplement au Miocène de la région paléarctique à partir de l'Afrique.

Fig. 17. - S. christophersi. Femelle : pharynx (a) ; cibarium (b) ; spermathèques (c). 


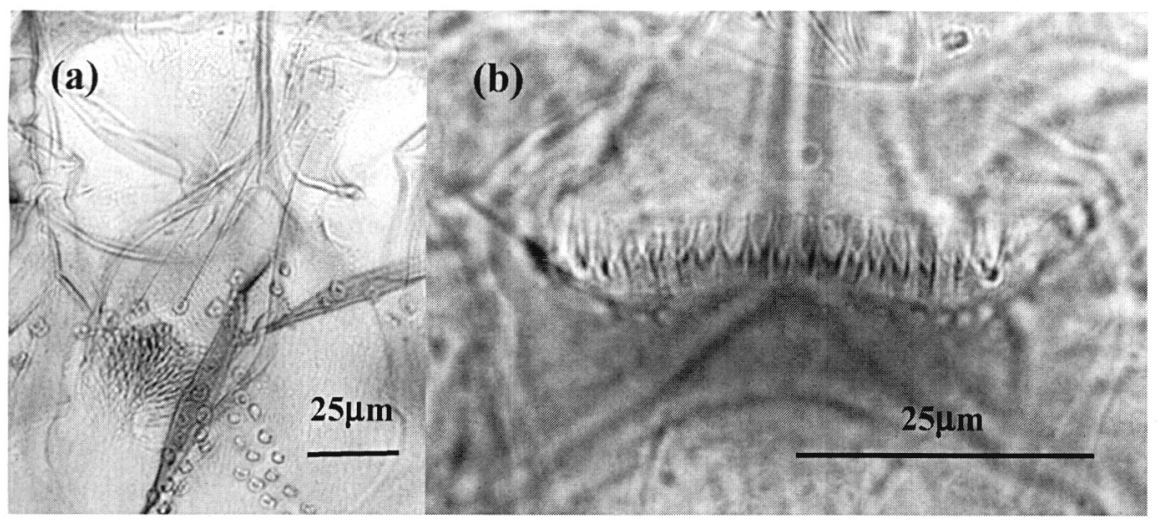

Fig. 18. - S. palestinensis. Femelle : pharynx (a) ; cibarium (b).

L'espèce de loin la mieux représentée est $S$. dentata, un phlébotome herpétophile constituant plus de $98 \%$ des captures. Les espèces zooanthropophiles du genre Phlebotomus sont peu abondantes, probablement à cause de l'utilisation généralisée des insecticides domiciliaires et agricoles. La faible densité des vecteurs prouvés ou suspectés de leishmaniose viscérale ( $P$. syriacus, $P$. tobbi) et cutanée anthroponotique ( $P$. sergenti) et zoonotique ( $P$. papatasi) explique le caractère rare et sporadique des cas de leishmanioses humaines au Liban.

Cependant, l'analyse de la distribution géographique de ces vecteurs fait apparaitre l'existence de deux foyers potentiels, l'un de leishmaniose viscérale (régions humides et semi-arides de faible et moyenne altitude du versant ouest du Mont Liban et moitié sud de la plaine de la Bekaa), l'autre de leishmaniose cutanée à L. major et L. tropica (zones arides du nord de la Bekaa).

Il est donc recommandé d'installer un système de surveillance régulière afin de détecter toute pullulation des vecteurs à la faveur d'un changement climatique ou de modifications éthologiques.

\section{REMERCIEMENTS}

N ous remercions la Coopération française au Liban ainsi que le Ministère français des Affaires étrangères pour avoir financé cette étude. Nous remercions également le professeur Bernadette AbiSaleh pour son aide matérielle qui a rendu possible la réalisation de ce travail.

\section{RÉFÉRENCES}

Al Zahrani M.A., Peters W., Evans D.A., Chin C., Smith V. \& LANE R. Phlebotomus sergenti, a vector of Leishmania tropica in Saudi Arabia. Transactions of the Royal Society of Tropical Medicine and Hygiene,1988, 82, 416.

Artemiev M.M. A revision of sandflies of the subgenus Adlerius (Diptera, Phlebotominae, Phlebotomus) .Zoologicheskiy Zhurnal, 1980, 59, 1177-1192 (en russe)
Artemiev M.M. \& Neronov V. Distribution and ecology of sandflies of the world (genus Phlebotomus). Inst. Evol. Morph. Anim. Ecol., Moscou, URSS, 1984, 208 p. (en russe).

BitAR J. \& NACHMAN H. Kala azar in Lebanon. Lebanese Medical Journal, 1964, 17, 257-268.

Buttiker W. \& Lewis D.J. Insects of Saudi Arabia. Some ecological aspects of Saudi Arabian phlebotomine sandflies (Diptera: Psychodidae). Fauna of Saudi Arabia, 1983, 5 , 479-528.

Croset H., Rioux J.A., Maistre M. \& Bayar N. Les phlébotomes de Tunisie (Diptera: Phlebotomidae). Mise au point systématique, chorologique et éthologique. Annales de Parasitologie humaine et comparée, 1978, 53, 711-749.

Dancesco P. Données sur la sous-espèce Phlebotomus chinensis balcanicus Theodor, 1958 (Diptera: Psychodidae) en Roumanie. Archives de l'Institut Pasteur de Tunis, 1968, 45, 185-194.

DePaquit J. Révision du sous-genre Paraphlebotomus (Phlebotomus: Phlebotominae: Diptera). Approches morphologique et moléculaire. Thèse, Université de Reims, Pharmacie, 1997, n² 211.

Depaquit J., Léger N., Ferté H., Rioux J.A., Gantier J.C., MichaeLIDES A. \& ECONOMides P. Les phlébotomes de l'île de Chypre. III - Inventaire faunistique. Parasite, 2001, 8, 11-20.

Doha S. \& Shehata M.G. Leishmania infantum MON-98 isolated from naturally infected Phlebotomus langeroni (Diptera: Psychodidae) in El Agamy, Egypt. Journal of Medical Entomology, 1992, 29, 891-893.

Guan L.R., Xu Y.X., Li B.S. \& Dong J. The role of Phlebotomus alexandri Sinton, 1928 in the transmission of kalaazar. Bulletin of the World Health Organisation, 1986, 64, 107-112.

Guerbouj S., Guizani I., De Joncker S., Dujardin J.C. \& NuWAYRI-SALTI N. Identification of Lebanese dermotropic putative Leishmania archibaldi isolates by gp63 PCRRFLP. Transactions of the Royal Society of Tropical Medicine and Hygiene, 2001, 95 (6) ,687-688.

Ismail M.T. \& Pesson B. Contribution à l'étude des phlébotomes de Syrie. Bulletin de la Société de Pathologie Exotique, 1992, 85, 317-321.

Javadian E., Mesghali A. \& Nadim A. Natural leptomonad infection of sandflies, with its first occurrence in $P$. alexan- 
dri in Khuzestan province, Iran. In : Écologie des leishmanioses. Colloque international du CNRS, Paris , 1977, 239, 203-205.

Killick-Kendrick R., Tang Y., Killick-Kendrick M., Sang D.K., Sirdar M.K., Ke L., Ashford R.W., Schorscher J. \& Johnson R.H. The identification of female sandflies of the sub-genus Larroussius by the morphology of the spermathecal ducts. Parassitologia, 1991, 33 (Suppl. 1), 335-347.

Knio K.N., BAYdoun E., TAWK R. \& NuWAYRi-SALTi N. Izoenzyme characterization of Leishmania isolates from Lebanon and Syria. American Journal of Tropical Medicine and Hygiene, 2000, 63 (1-2), 43-47.

Léger N., Depaquit J., Ferté H., Rioux J.A., Gantier J.C., Gramiccia M., Ludovisi A., Michaelides A., Christophi N. \& EcoNOMides P. Les phlébotomes de l'île de Chypre. II - Présence de Leishmania (Leishmania) infantum Nicolle, 1908 (zymodème MON 1) chez Pblebotomus (Larroussius) tobbi Adler et Theodor, 1930. Parasite, 2000, 7, 143-146.

Léger N., Gramiccia M., Gradoni L., Madulo-Leblond G., Pesson B., Ferte H., Boulanger N., Killick-Kendrick R. \& KILLICK-KENDRICK M. Isolation and typing of Leishmania infantum from Phlebotomus neglectus on the island of Corfu, Greece. Transactions of the Royal Society of Tropical Medicine and Hygiene, 1988, 82, 419-420.

Léger N., Haddad N. \& Chaker A. Description de Phlebotomus (Synphlebotomus) saltiae n. sp. (Diptera: Psychodidae) du Liban. Bulletin de la Société de Pathologie Exotique, 1997. 90, 55-58.

Léger N., Nuwayri Salti N., Abou-Fakhr E., Sadek R. A., SheBAKLO Z. \& LaKkis M. Les phlébotomes du Liban. I. Résultats de récoltes préliminaires (août 1994). Parasite, 1995 , 2, 93-94.

LÉger N. \& Pesson B. Sur la taxonomie et la répartition géographique de Phlebotomus (Adlerius) chinensis s. 1. et de P. (Larroussius) major s. 1. (Psychodidae-Diptera). Bulletin de la Société de Pathologie Exotique, 1987, 80, 252-260.

LÉGer N., Pesson B. \& Madulo-Leblond G. Les phlébotomes de Grèce. $1^{\text {ère }}$ partie. Bulletin de la Société de Pathologie Exotique, 1986a, 79, 386-397.

Madulo-Leblond G. Les phlébotomes (Diptera: Phlebotomidae) des Iles Ioniennes. Thèse d'Université, Sciences pharmaceutiques, Reims, 1983, $\mathrm{n}^{\circ} 4$.

Nuwayri-Salti N., Baydoun E., Alema-Munoz M.M. \& KreutZER R. Identification of Leishmania isolates from a Lebanese population. American Journal of Tropical Medicine and Hygiene, 1994, 51, 98-101.

Nuwayri-Salti N., Baydoun E., El-Tawk R., Fakhouri Makki R. \& KNIO K. The epidemiology of leishmaniases in Lebanon. Transactions of the Royal Society of Tropical Medicine and Hygiene, 2000, 94 (2), 164-166.

Nuwayri-Salti N., Nasr R., Haddad K., Chamat S. \& Usta J. Canine leishmaniasis in northern Lebanon. Annals of Tropical Medicine and Parasitology, 1997, 91, 221-222.

Perrotey S., Benabdennbi I., Haddad N., Pesson B. \& Léger N. Electrophoretic and morphological differentiation between two sympatric species of Adlerius: Pblebotomus brevis and Phlebotomus simici (Diptera: Psychodidae). Journal of Medical Entomology, 2000, 37 (2), 289-294.
Rioux J.A., Léger N., Haddad N., Gramiccia M., Jalouk L., Dereure J., Al-Khiami A. \& Desjeux P. Infestation naturelle de Phlebotomus tobbi (Diptera, Psychodidae) par Leishmania donovani s. st. (Kinetoplastida, Trypanosomatidae), en Syrie. Parassitologia, 1998, 40 (suppl. 1), 148.

THEODOR O. On some sandflies (Phlebotomus) of the sergenti group in Palestine. Bulletin of Entomological Research, 1947, 38, 91-98.

Theodor O. \& Mesghali A. On the Phlebotominae of Iran. Journal of Medical Entomology, 1964, 1, 285-300.

Reçu le 18 septembre 2002 Accepté le 10 janvier 2003 